\title{
A pele do recém-nascido prematuro sob a a avaliação do enfermeiro: cuidado norteando a manutenção da integridade cutânea
}

The skin of the premature newborn under the nurse's evaluation: orientating the manteinance of cutaneous integrity

La piel del recién nacido prematuro bajo la evaluación del enfermero: el cuidado direccionado para la manutención de la integridad cutánea

\section{Christiane Pereira Martins', Carmen Elisa Villalobos Tapia"}

'Universidade de São Paulo. Escola de Enfermagem. São Paulo, SP

"Pontifícia Universidade Católica de São Paulo. Faculdade de Enfermagem. São Paulo, SP

Submissão: $12 / 12 / 2008$

Aprovação: $31 / 08 / 2009$

\section{RESUMO}

A prematuridade expressa relevância à saúde neonatal, demonstrando comprometimento ascendente com relação a morbimortalidade desta clientela, cuja maior incidência pode ser atribuída às práticas inadeQuadas com a pele do prematuro. Revisão da literatura sobre a interação benéfica entre a pele do Recém-Nascido Pré-Termo e o manuseio adeQuado, norteado pelo enfermeiro, Que por meio da padronização dos cuidados de interferência direta e avaliação contínua da integridade cutânea e o assistir o prematuro. Divulga a importância do cuidado com a pele do Recém Nascido Prematuro, possibilitando não apenas a sobrevivência, mas a oportunidade de se integrar e desfrutar da vida com Qualidade.

Descritores: Recém-nascido; Enfermagem; Assistência de enfermagem.

\section{ABSTRACT}

The prematurity expressed relevance to the health neonatal, demonstrating ascending compromising regarding morbi-mortality of this patients, whose larger incidence can be attributed to the inadequate practices with the skin of the premature. Revision of the literature about the beneficial interaction among the skin of premature infant newborn and the appropriate handling, orientated by the nurse, that through the standardization of the cares of direct interference and continuous evaluation of the cutaneous integrity and attending the premature. It publishes the importance of the care with the skin of the premature newbor, not just making possible the survival, but the opportunity to integrate and to enjoy of the life with Quality.

Descriptores: Infant, newborn; Nursing; Nursing care.

\section{RESUMEN}

La prematuridad expresa una relevancia para la salud neonatal, demonstando un comprometimiento ascendiente la relación de la morbimortalidade de esta clientela, cuya mayor incidencia puede ser atribuida a las prácticas inadecuadas con la piel del prematuro. Revisión de la literatura sobre la interacción beneficiosa entre la piel del recién nacido pré-termino y el manejo adecuado direccionado por el enfermero, Que por medio de la padronización de los cuidados de interferencia directa y evaluación continua de la integridad cutánea y la asistencia al prematuro. Divulga la importancia del cuidado con la piel del recién nacido prematuro, posibilitando no apenas la sobreviviencía, como la oportunidad de integrar y disfrutar de la vida con cualidad

Descriptores: Recién nacido; Enfermería; Atención de enfermería. 


\section{INTRODUÇÃO}

O nascimento inicia-se com a expulsão do bebê, seguida pelo clampeamento e secção do cordão umbilical, estas ações produzem significativas alterações circulatórias Que, comparadas ao choro e a expansão dos pulmões, causam em Questão de segundos, a adaptação do recém nascido (RN) a um novo ambiente ${ }^{(1)}$. Recémnascido é o concepto expulso do organismo materno, e classificao como normal ou termo, aquele com idade gestacional (IG) de trinta e sete semanas a Quarenta e uma semanas e seis dias (de 259 a 293 dias) ${ }^{(2)}$.

O recém-nascido deve ser classificado por IG e peso ao nascer, estes parâmetros devem ser avaliados no gráfico de crescimento intra-uterino do Colorado ${ }^{(3)}$ é o mais utilizado atualmente, e propicia a classificação dos RNs. Dessa forma, após pesar o recém nascido e determinar a idade gestacional, estes dois dados são analisados no gráfico. O recém nascido cujo peso estiver entre o percentil $10^{\circ}$ e $90^{\circ}$ é classificado como adequado à IG, e aquele cujo peso estiver fora desta faixa é considerado pequeno ou grande para a I.G., respectivamente, abaixo do percentil $10^{\circ}$ e acima do percentil $90^{\mathrm{o}(4)}$. Com este instrumento pode-se classificar o concepto pela I.G., isoladamente ${ }^{(4)}$ :

Pré-termo (PT): IG < 37 semanas.

Termo (T): IG entre 37 e 41 semanas

Pós-termo (PoT): IG > 42 semanas

Define-se prematura, a criança nascida antes do tempo de gestação(5) ${ }^{(5)}$ o recém nascido pré termo (RNPT) é conceituado pela Organização Mundial de Saúde, como aquele nascido antes do tempo, com IG inferior a trinta e sete semanas ${ }^{(6)}$.

Os recém-nascidos prematuros possuem características físicas e neurológicas Que são diferentes em cada estágio de desenvolvimento, cuja identificação destas, a partir do Método de Capurro e exame neurológico, fornece dados valiosos para a IG e capacidades fisiológicas dos recém nascidos ${ }^{(7)}$. Dessa forma, o RN avaliado prematuro ou PT é encaminhado a Unidade de Terapia Intensiva Neonatal (UTIN) $)^{(4)}$. Sabemos Que nas UTINs, o RNPT necessitará de recursos materiais e procedimentos invasivos Que fornecerão subsídios à sua sobrevivência. Dentre estes recursos e procedimentos, serão considerados no presente estudo aqueles em Que a pele esteja envolvida.

As práticas de cuidados com a pele realizada diariamente nas UTIs Neonatais incluem a manutenção da temperatura e umidade do ambiente, por meio de incubadoras, o posicionamento, o banho, a lubrificação com óleos emolientes, o uso de soluções cutâneas para anti-sepsia, fixação ou remoção de adesivos para suporte à vida e aparelhos de monitorização, realização de procedimentos invasivos, como punções venosas ou arteriais ${ }^{(8)}$.

O manuseio apropriado da pele do RNPT denota a valorização, pelo enfermeiro e sua equipe, dos detalhes inerentes nos cuidados prestados. É uma prática desafiadora, porém bela, responsável por repercussões no sistema de vida desses recém nascidos prematuros, ou melhor, pela Qualidade de vida.

Tendo em vista a importância deste tegumento para a sobrevivência do prematuro, estratégias têm sido realizadas para manter a integridade da pele, para tanto, há necessidade de padronização dos cuidados de enfermagem, e Que esta esteja presente na prescrição de enfermagem de cada RN, tornando possível a individualização do cuidado prestado à pele do prematuro.

\section{MÉTODO}

\section{Tipo de Estudo}

O estudo bibliográfico realizado com materiais publicados em periódicos e livros nacionais e internacionais indexados, dos Quais se procurou compreender a realidade estudada, publicados nos Quinze últimos anos.

\section{Coleta de Dados}

A coleta de dados foi realizada por meio da busca manual e com o uso de ferramentas da informática nas Bibliotecas da PUCCampinas e Unicamp. Em sites disponíveis na internet, com a realização de um levantamento dos artigos "on-line" na BIREME (Centro Latino Americano e do Caribe em Ciências da Saúde) e base de dados LILACS (Literatura Latino-Americana e do Caribe em Ciências da Saúde) por meio dos termos de indexação.

\section{Análise dos Dados}

Após a coleta de dados, foi realizada de forma comparativa a partir das necessidades do estudo, apresentando em forma de Quadro o referencial bibliográfico levantado, para posteriormente iniciar a análise dos textos.

\section{RESULTADOS}

Por meio da coleta de dados foram recuperados um artigo relacionado à iniciação científica da FAPESP, um artigo publicado em site, Quinze livros, oito periódicos e duas monografias; entretanto, efetivamente foram trabalhados com os dados garimpados em seis livros, seis periódicos, duas monografias e um artigo publicado em site.

Constatamos unanimidade na conceituação do recém nascido prematuro, como aQuele nascido antes do tempo, apresentando IG inferior a trinta e sete semanas.

Os autores pesquisados concordam e garantem que muitos aspectos relacionados aos cuidados com o RN são alterados por diversas vezes; porém a participação familiar associada a avaliação comportamental dos RNs, vêem contribuindo à neonatologia, trazendo uma assistência sob nova ótica, Que exige desta ciência novos conhecimentos, aprimoramento, desafio nas intervenções, e a desmistificação do diferente e do novo.

$\mathrm{Na}$ unidade neonatal, meio extra-uterino, o neonato pré-termo vivencia diversas experiências, cujas deveriam ter sido experimentadas e desenvolvidas ainda intra-útero, e neste contexto para todos os autores, torna-se imprescindível à estimulação e intervenção ao RNPT, para Que haja interferência direta no Sistema Nervoso Central (SNC), e conseqüentemente maturação neurológica.

O primeiro sistema orgânico a se desenvolver é o tátil, é o sentido de maturação mais precoce, Que permite reconhecimento e reação diante dos diferentes tipos de toque, função esta Que possibilita o aprendizado, a maturação neurológica ${ }^{(9)}$.

Neste estudo os autores caracterizam a pele do prematuro como tegumento de barreira contra infecções, sendo a primeira linha de defesa. Ao toque a pele do RNPT é Quente, úmida e aveludada 
(lanugem), possui uma estrutura sensível, caracterizada pele presença de estrato córneo (camada cutânea mais externa da pele do $\mathrm{RN}$ ) delgado e hipodesenvolvido, porém hidratado por uma substância denominada vérnix, Que se encontra sobre a superfície cutânea ao nascimento ${ }^{(8,9)}$.

Apuramos dados significativos em relação a fragilidade deste tegumento, tais como a percentagem de $13 \%$ da superfície corporal Que a pele representa ao nascimento, o risco para instabilidade térmica, perda de calor, aumento das necessidades hídricas, maior absorção transepidermica se substâncias aplicadas sobre a pele, maior colonização e infecção invasiva. Pela imaturidade funcional da pele associada ao manejo inadequado dos profissionais, temos aproximadamente $80 \%$ de morbimortalidade relacionada ao fator pele (traumas ou alterações da função normal) ${ }^{(8,10)}$.

As principais funções de pele descritas pelos autores referendados referem-se a modulação do fluxo de água transepidermal, termorregulação, defesa antimicrobiana, proteção contra toxinas do meio externo e função tátil; estas funções atingem total maturação entre 2-4 semanas, podendo atingir oito semanas nos RNPT extremos.

A temática sobre a pele do RN prematuro é conduzida com maior profundidade, considerando o RNPT como sujeito, possibilitando-o vivenciar experiências de aprendizado e de troca com o meio, desafiando a ciência esta interface psicológica vem demonstrando, gradativamente, resultados fisiológicos e comportamentais bastante expressivos ${ }^{(9,11)}$.

Neste contexto conclui-se Que a pele é o órgão do sentido mais amplo do corpo e importante para a sobrevivência do ser, sendo por meio deste tegumento Que os RNs percebem e conhecem o mundo, por isso a relevância do início da experiência tátil, entretanto retoma Que antecede o toQue a reflexão sobre o motivo e qualidade do contato a ser oferecido ${ }^{(1)}$. Os RNs prematuros permanecem por um período, muitas vezes prolongado, nas UTIN., sendo nesta unidade Que estes prematuros recebem as primeiras experiências táteis, Que em sua maioria estão associadas a procedimentos técnicos.

Dentre as práticas de cuidados com a pele do RNPT, comentados anteriormente, segue a análise individual de cada prática, associada as principais alternativas para a rotina estabelecida nas UTIN, possibilitando aos profissionais melhor manejo com o RN prematuro, tornando-o participante a cada cuidado.

Com relação à manutenção da temperatura corporal, os autores consultados apontaram Que embora o RNPT possua o centro da regulação térmica, este pode ser total ou parcialmente inativado por várias drogas e alterações patológicas. Esta imperfeição no controle da temperatura também está relacionada com a superfície corporal relativamente grande em relação ao peso, menor isolamento térmico e a presença de peQuena massa para produção, regulação e manutenção da temperatura.

A manutenção da termoneutralidade em prematuros é possível por meio de aparelhos, neste caso incubadoras, onde o uso deste artifício é destinado aos RNPT. com peso de nascimento inferior a 1800 gramas, acrescenta a preferência de incubadoras de dupla parede com umidificação para os RNPT com peso inferior a 1000 gramas $^{(10)}$.

Ambiente térmico neutro é aquele Que permite que o RN mantenha temperatura interna normal, com consumo mínimo de oxigênio e energia, propiciando ao prematuro, Que apresenta a mínima camada de gordura subcutânea, controle a perda ou ganho de calor corporal somente dentro de uma faixa limitada de temperatura ambiente. Torna-se extremamente complicado precisar a termoneutralidade ideal, porém consolidamos que para oferecer um ambiente térmico ideal a temperatura axilar do RN deverá oscilar dentro de uma faixa de $36.5^{\circ} \mathrm{C}$ a $37.5^{\circ} \mathrm{C}^{(12)}$.

Recomendamos o mínimo manuseio, devido o R.N. prematuro apresentar dificuldade em conservar calor com mudanças posturais, podendo alternar o decúbito de $4 / 4$ horas, porém o rigor da freQüência deverá ser respeitado no cuidado de prematuros sedados ou Que apresentarem baixa atividade motora. Recomenda manter o R.N. aninhado no leito, sempre Que possível, em posição fetal; minimizando a transferência de calor da superfície corporal para o meio ambiente, como mecanismo fisiológico ${ }^{(10)}$.

A perda de água transepidermal é o principal meio de perda de calor em RN prematuros; por isso salientamos a importância do controle da UR do ar, Que conforme os autores deve ser superior a $75 \%{ }^{(8,9)}$.

Acrescentamos Que a temperatura da incubadora deve ser mantida na zona termo neutro, controlando regularmente a temperatura corporal do RN, Que será exposto durante os primeiro 07 dias de vida a UR de $85 \%$, podendo reduzir após este período a percentagem, mantendo nas primeiras três semanas de vida a uma UR interna da incubadora de $70-75 \%$, sendo reduzida gradualmente depois deste período até $60 \%$, conservando nesta percentagem até o alcance de peso, nos RNPT baixo peso, 1500 gramas. Os gases ofertados ao RN também deverão encontrar-se em temperatura e umidade satisfatórias ${ }^{(13)}$.

O posicionamento do RN é uma outra estratégia utilizada para reduzir a perda térmica, pois além de desenvolver parâmetros fisiológicos e comportamentais, mantêm o tônus muscular mais adequado, possibilita padrões normais de movimento, reduz contraturas e deformidades, e por fim gera mais conforto e segurança. A diminuição da perda de calor por meio de contenção postural ocorre devido a redução da superfície da corporal do RN e seu contato com objetos termicamente neutros, como tecidos de algodão $0^{(14)}$.

O uso de óleos, tais como Petrolato, Girassol e Canola, na rotina das UTIN, demonstram auxílio na funcionalidade da pele do prematuro. A aplicação de óleo duas vezes por dia nas primeiras duas semanas de vida (período Que ocorre a maturação da camada mais externa da pele, o extrato córneo) na pele do RNPT com IG inferior a 33 semanas de vida, momento Que inicia o desenvolvimento desta camada, revelam resultados expressivos, como a redução dos episódios de sepse e incidência de meningite ${ }^{(8)}$.

A autora ainda descreve os benefícios da aplicação tópica de óleos, sendo estes a proteção da integridade do extrato córneo, reforçando a função de barreira da pele, aumento da hidratação da pele e diminuição de infecções em prematuros, melhora da nutrição por meio da absorção percutânea de lipídeos, equilíbrio da PTE A e melhora do desenvolvimento neurológico, promovendo o contato mãe-bebê pelo estímulo tátil da aplicação.

Referente ao banho, procedimento diário e de rotina nas UTIN, consideramos ser um procedimento desnecessário, e contra indicado em R.N. prematuros. Asseguramos Que há necessidade de higienizar somente a área dos genitais, com água morna e sabão neutro, porém 
nos R.N.s com peso inferior a 1500 gramas, a higiene deve ser efetuada somente com água, pois nestes RNs a fragilidade da pele, principalmente no fator nutrição/hidratação, é considerada duas vezes maior que a de RN com peso de 1800 gramas. Com este cuidado, garantiremos a manutenção da integridade da pele, deixando inalteradas suas propriedades de barreira (manto ácido), a termorregulação, funções metabólicas e controle dos dados vitais ${ }^{(10)}$.

$\mathrm{O}$ autor ainda salienta Que o banho deve ser prorrogado no mínimo até o RNPT atingir duas semanas de vida, e depois deste período, realizá-lo por tempo curto menor que cinco minutos, no ambiente da incubadora, restringindo-se a área suja e de forma infrequente (menor Que três vezes por semana).

Sugerimos o clorexidine como alternativa, por ser eficaz na redução da colonização da pele por um período de Quatro até seis horas após o banho e não haver descrição científica de toxicidade, embora ocorra absorção ${ }^{(8)}$. A maturidade da pele determina o grau de absorção percutânea, e em prematuros, a pele, logo ao nascimento, apresenta comprometimento pela imaturidade, facilitando, portanto a absorção de substâncias aplicadas na superfície da pele.

A descontaminação da pele do prematuro antes de procedimentos invasivos é freeüente na rotina das UTIN, pela necessidade de reduzir a colonização da pele, fator importante e de risco para a infecção sistêmica, principalmente Staphylococcus, microorganismo determinante de sepse em RNPT. ${ }^{(8)}$.

Portanto, como alternativa para obtenção de um amplo espectro de antissepsia da pele do RNPT. à procedimentos invasivos dos mais diversos sem comprometer o tegumento, a aplicação tópica de clorexidine $0.5 \%$ sendo duas exposições e intervalo de 10 segundos ou uma exposição de 30 segundos, havendo necessidade de retirar o resíduo (excesso) do produto com gaze estéril embebida em água destilada estéril ou soro fisiológico $0,9 \%(8,10)$.

As punções venosas e arteriais compõem a rotina a Que os RNs são impostos nas unidades neonatais. Indicamos o agrupamento das coletas de sangue, a fim de evitar tais procedimentos e minimizar e/ou prevenir a dor, Que frente à freqüência destas experiências ocasiona-se hiperinervação e hiperalgesia da área perfurada por proliferação das fibras nervosas na reparação deste local ${ }^{(14)}$.

A hidratação adequada é de particular importância em RNPT uma vez Que o conteúdo de água extracelular deles é maior (70\% em RNT e até $90 \%$ em RN prematuros), portanto, há necessidade de haver acesso vascular para a administração de fluidos parenterais suplementares, Que oferecem caloria adicional, eletrólitos e/ou água, evitando a depleção hídrica e comprometimento cutâneo oriundo deste desequilíbrio ${ }^{(7)}$.

Torna-se essencial Que o primeiro acesso vascular seja o umbilical (venoso e arterial), sendo possível Que estas coletas de sangue sejam realizadas por meio deste acesso. A cateterização umbilical pode ser procedida logo após o nascimento, permanecendo de três até sete dias pós-natal (dependendo do material Que o cateter tenha sido feito), Quando se deve acessar novo vaso por meio de cateter central de inserção periférica (PICC), de preferência Que apresente mais de um lúmen, assim proporcionando a continuidade da proposta supra comentada. Ressalta Que, em casos de não haver possibilidade de instalação do P.I.C.C. por inviabilidade vascular, poderá ser mantido o cateter umbilical até no máximo 10 dias. Na impossibilidade de acesso venoso, proceder apenas acesso arterial, e/ou vice-versa, mantendo a viabilidade do cateter por meio da utilização de heparina nas soluções de infusão, preservando assim as investidas na pele do $\mathrm{RN}$ no intuito de se conseguir acesso venoso vascular. Descarta-se, portanto a coleta de amostras de sangue via punção do calcanhar ${ }^{(13)}$.

O autor supra citado ressalta Que a utilização de cateteres periféricos, como "Jelco", deverão ser reservados para casos especiais em Que não se consegue acesso profundo ou para transfusão de hemoderivados.

A implementação do PICC inova a terapêutica via parenteral, contribuindo imensamente na redução do desgaste (estresse) do R.N., de sua família e de toda a equipe multiprofissional, Que se encontra envolvida na manutenção do acesso venoso, e neste caso igualmente, a integridade da pele.

Com a utilização deste cateter central, o RN prematuro terá menor risco de infecção por ser manuseado minimamente, reduzido exposição a múltiplas punções vasculares e a diminuição bastante significativa das dissecções venosas; além de propiciar menos estresse, contribuindo ao desenvolvimento do SNC, diminuição das experiências dolorosas, desconforto, lesões cutâneas, providas muitas vezes de infiltração de soluções, preservação pilosa do RN e aumento da Qualidade de vida durante o período de internação na UTIN ${ }^{(13)}$.

Contudo, o uso de cateteres central, significa uma solução para diversos fatores previamente contemplados e uma solução de continuidade na área inserida, dessa forma estabelece o risco para colonização deste cateter demandando um cuidado criterioso tanto no momento de sua inserção Quanto nos cuidados diários viabilizados pelos profissionais Que o manipulam, conforme a padronização da comissão de controle de infecção hospitalar (CCIH) de cada instituição.

A fixação dos dispositivos utilizados na monitorização e permanência de cateteres em prematuros deve ser efetuada de maneira discriminada e cautelosa Quando a indicação estiver estabelecida.

De modo geral, os autores referendados indicam o micropore como fonte adesiva destes materiais, porém este adesivo ou QualQuer outro Que possua maior potencial de aderência deve ser empregado sobre fina cobertura de hidrocolóide ou filme transparente previamente aplicado na pele do prematuro, ao uso de cateteres vasculares, sondas, tubo endotraqueal, dispositivo plástico de coleta urinária. No caso de fixação de cateteres vasculares, a fixação deve ser efetuada com cobertura adesiva transparente, antialérgica, favorável a permeabilidade gasosa e impermeável a contaminantes externos, propiciando avaliação contínua do local de inserção, Quanto presença de sinais flogísticos e infiltração, sobretudo havendo menor manipulação. A troca desta película deve ser realizada no mínimo a cada sete dias ${ }^{(13)}$.

Assim, apontamos alternativa para fixação do sensor do oxímetro e proteção ocular do RN exposto a fototerapia, o uso de tiras a pressão auto-adesiva com peças de velcro desenvolvidas para tal, para tanto se torna indispensável manter a vigilância para Que a posição do mesmo não seja fator de lesão de pele ${ }^{(13)}$. Sugerimos Que se alterne o local de fixação, em média, a cada 4 horas, considerando a individualidade e características da pele de cada prematuro para determinar a freqüência do rodízio, como profilaxia de comprometimento cutâneo e dor, garantindo a circulação 
sanguínea do local. Ressalta ainda Que o sensor do oxímetro de pulso deva ser fixado com firmeza, porém de maneira confortável, descomprometendo a movimentação do $\mathrm{RN}^{(14)}$.

Com relação ao uso de dispositivo plástico de coleta urinária, recomenda-se a aplicação de bolas de algodão sobre a genitália do prematuro ou na fralda descartável do mesmo, realizando posteriormente manobra de expressão, em um saco plástico, na bola de algodão embebida de diurese, possibilitando a aspiração deste fluido com uma seringa. Este é o meio mais simples, eficaz e menos traumático de se aferir o débito e controle urinário; embora seja comentado o uso da pesagem da fralda descartável, convertendo diretamente o peso em mililitros (por ex., $25 \mathrm{~g}=25 \mathrm{ml}$ ), consideramos Que este último método garante somente o registro do volume urinário eliminado ${ }^{(15)}$.

Os adesivos devem ser mantidos na pele por pelo menos 24 horas, exceto na necessidade de refixação por despreendimento do adesivo anterior ou data de validade do material, como sonda gástrica. Destaca Que os eletrodos de monitor cardíaco demandam remoção somente Quando não estiverem funcionantes, e na certeza de Que estes não serão mais necessários ${ }^{(10)}$.

Para a remoção dos adesivos, recomendamos cotonetes embebidos em óleo mineral, tal prática têm sido bastante explorada nas UTIN $^{(7)}$. Contudo, o uso de solventes, comumente encontrados e utilizados por estas unidades na remoção adesiva, é contraindicado pelo risco de absorção e toxicidade, assim como a aplicação tópica de tintura de beinjoim, Que acrescenta o risco de lesão da pele na retirada do adesivo ${ }^{(10)}$.

A prevenção implica uma série de "detalhes", simples de realizar, mas difíceis de lembrar, assim, instalada a lesão na pele do prematuro, decorrente muitas vezes da escassa paciência do profissional Que a manipula, portanto sugerimos a utilização de produtos apropriados no local da pele comprometida. Óleos á base de Petrolato (Aquafor ou Bacitracina) possuem amplo potencial cicatrizante, promovem uma cobertura semi-oclusiva na pele, permitindo a permeabilidade gasosa; tendo como resultado a migração dos Queratinócitos para o interior da matriz da ferida, evoluindo surpreendentemente após aproximadamente cinco dias do início da terapêutica ${ }^{(8)}$.

A autora supra citada sugere outros produtos alternativos, como o hidrocolóide, Que forma uma camada epitelial artificial, útil na profilaxia de traumas na pele do RNPT, Quando aplicado sobre a pele antes de fixar cateteres ou outros dispositivos, colaboram na repitelização e cicatrização da lesão da pele do prematuro.

Com o propósito de visar à manutenção da integridade da pele do prematuro, propondo alternativas simples, porém inovadoras para a prática tradicional, torna-se de suma importância a participação tanto do Enfermeiro Quanto de toda a equipe multiprofissional, para que esta proposta de cuidados e tratamentos possa ser devidamente desenvolvida, implementada e dada continuidade.

Este estudo revelou um momento de amadurecimento da assistência neonatal, em Que se compreende Que o tratamento do RN envolve muito mais do Que apenas a utilização de procedimentos e técnicas; a presença da família e o cuidado com o RN são aspectos Que demonstram notável diferença.

A existência de um protocolo sobre o manuseio da pele do prematuro permite avaliação periódica das características da pele do $\mathrm{RN}$, diagnosticando possibilidades profiláticas e de comprometimento cutâneo, como riscos de ruptura da pele, substâncias tópicas passíveis de alteração neste tegumento, desta forma as condutas elaboradas são contempladas na prescrição de enfermagem, expressando o caminho Que a equipe deve seguir ao assistir o RN prematuro no Que tange a sua pele.

\section{CONSIDERAÇÕES FINAIS}

Pela literatura identificada e analisada, percebemos que o desafio está lançado para cuidar do RN prematuro com esta nova ótica apontada neste estudo, Que visa atuar profilaticamente e/ou melhorar em curto prazo, as repercussões no SNC e na vida de relação desses prematuros. O conhecimento sobre o cuidado com a manipulação do prematuro e sua pele, e o limite de cada intervenção, possibilita à equipe uma nova estrutura, com mudanças no paradigma, Que verte a uma nova prática.

O estudo pretende agregar informações, em relação ao manuseio da pele do RNPT, ao Que a equipe já possui; visto que o contato com o RN prematuro é freqüente na UTIN, onde raramente estes RNs são deixados Quietos por mais de uma hora. Portanto apresentamos medidas relacionadas às ações e atitudes do profissional, Que exibe capacidade de propiciar melhora no padrão de manuseio da UTIN, desde a individualização do cuidado de acordo com o desenvolvimento e as capacidades do R.N., mantendo um equilíbrio entre contenção (aninhamento por meio de tecidos ou manual, desprovidas de pressão), fornecendo limites e suporte para o corpo, exploração e auto organização.

Consideramos Que cabe ao profissional enfermeiro indicar a direção adequada para o cuidado, por meio de um protocolo de manuseio da pele do RN prematuro, validar o cuidado (diária e periodicamente) e intervir Quando necessário. Por isso além do protocolo sugerimos a elaboração de um instrumento para avaliar diariamente a pele do prematuro, promovendo uma evolução descritiva deste tegumento, e permitindo o diagnóstico de QualQuer alteração nesta barreira e suas conseQüências.

A profusão de informações Que envolvem a assistência ao RNPT expressa um caminho a ser traçado por aQueles Que almejam Qualidade em suas ações.

\section{REFERÊNCIAS}

1. Siqueira AAF. O nascimento biológico da criança. Rev Bras Crescimento Desenvol Humano 1991; I(1): 44-53.

2. Jacomo AJD. Assistência ao Recém Nascido: Normas e Rotinas. São Paulo: Atheneu; 1998.

3. Battaglia FC, Lubchenco LO. A practical classification of newborn

infants by weight and gestacional age. I Pediatrics 1967; 71 : 159-63.

4. Kenner C. Enfermagem neonatal. Rio de Janeiro: Reichmann \& Affonso; 2001.

5. Ferreira $\mathrm{ABH}$. Minidicionário da Língua Portuguesa. Rio de 
Janeiro: Nova Fronteira; 1993.

6. Calil VMLT. Caracterização do recém-nascido pré-termo. In: Leone CR. Assistência integrada ao recém-nascido. São Paulo: Atheneu; 1996.

7. Whaley LF, Wong DL. Enfermagem pediátrica: elementos essenciais à intervenção efetiva. Rio de Janeiro: Guanabara Koogan; 1999.

8. Hahn LP. Pele do recém-nascido prematuro [monografia]. Curitiba: Departamento de Pediatria do Hospital de Clínicas, Universidade Federal do Paraná; 2001 .

9. Ministério da Saúde (BR). Manual Técnico Atenção Humanizada ao Recém-Nascido de Baixo Peso-Método Canguru / Secretaria de Políticas de Saúde, Área Saúde da Criança-Brasília: Ministério da Saúde; 2001 .

10. Darmstadt T, Dinulos JG. Neonatal skin care. Pediatr Clin North Am 2000; 47(4):757-82
11. Donice MG. A espiritualidade do contato "a massagem bioenergética neonatal de Eva Reich, como promoção da saúde e como prevenção da biopatia". Rev Intern Anima Corpo Psicol Somática 1997.

12. Thomas K. Thermoregulation in neonates. Neonatal Network 1994; 13(2): 15-22.

13. Margotto PR. Recém-nascido pré-termo extremo-limite de viabilidade. Brasília; 200l. Disponível em: http:// www.medico.org.br

14. Zaconeta CM. Neonatologia, a terceira onda [monografia]. Brasília: Departamento de Terapia Intensiva Neonatal do Hospital Materno Infantil de Brasília; 2001.

15. Fox MD. Measurement of urine output volume: accuracy of diaper weights in neonatal environments. Neonatal Network 1992; 11(3):11-8. 